Author copy of paper, accepted and published in:

Macromolecular Theory and Simulations 20, 496 - 509 (2011)

The final published copy of the article is available at http://onlinelibrary.wiley.com/doi/10.1002/mats.201100015

\title{
Two-dimensional Wang-Landau algorithm for osmotic pressure calculations in a polyelectrolyte-membrane system
}

\author{
Nikolay A. Volkov* \\ Division of Physical Chemistry, Department of Materials and Environmental Chemistry, \\ Stockholm University, S-106 91, Stockholm, Sweden \\ Pavel N. Vorontsov-Velyaminov ${ }^{\dagger}$ \\ Faculty of Physics, St. Petersburg State University, 198504, St. Petersburg, Russia \\ Alexander P. Lyubartsev ${ }^{\ddagger}$ \\ Division of Physical Chemistry, Department of Materials and Environmental Chemistry, \\ Stockholm University, S-106 91, Stockholm, Sweden
}




\begin{abstract}
The Monte Carlo method based on two-dimensional entropic sampling within the Wang-Landau (WL) algorithm is applied to simulation of a continuous model of a polyelectrolyte between membrane surfaces. Membranes are presented by parallel plane surfaces holding either fixed or mobile dipoles (representing lipid headgroups). A strongly charged polyion accompanied by neutralizing counterions is placed between the membranes. Periodic boundary conditions are imposed along $\mathrm{X}$ and $\mathrm{Y}$ axes. The volume of the main cell is varied during the simulation by shifting one of the surfaces along Z-axis. Within two-dimensional WL sampling algorithm we obtain joint density of states as a function of energy and volume in a single run. In order to increase efficiency of our calculations we introduce a number of modifications to the original WL-approach. Various properties of the system over wide temperature and volume or pressure ranges, i.e. conformational energy, heat capacity, free energy, are obtained from the two-dimensional density of states by simple integration. The osmotic pressure is calculated as a derivative of Helmholtz free energy. Alternatively, properties of the system, including average volume, can be obtained under condition of NPT ensemble. It is shown that the both approaches produce coinciding $P_{o s m}(V)$ isotherms. In all considered cases we observe repulsive effective interaction between the membrane surfaces, and repulsion is stronger for the surfaces with fixed dipoles.
\end{abstract}

*Electronic address: Nikolay.Volkoff@gmail.com

${ }^{\dagger}$ Electronic address: voron.wgroup@gmail.com

${ }^{\ddagger}$ Electronic address: alexander.lyubartsev@mmk.su.se 


\section{INTRODUCTION}

Interactions of various macromolecules with biomembranes are an essential part of many important cellular processes. A thorough understanding of these interactions is one of the greatest challenges in biophysical and biomedical sciences. In particular, the detailed information regarding such highly heterogeneous molecular systems and their components is extremely desirable for an effective and safe use of various oligomer and polymer (polyelectrolyte) chains in biomedical applications. The properties of biomembrane assemblies are not of only biological and biomedical, but also of biotechnological and environmental relevance, for instance in purification or catalysis processes.

Biological membranes are composed of a complex array of various lipid molecules. Membrane lipids, being organized into a bilayer structure, serve as a basic matrix for other constituents of biomembranes. Therefore, lipid bilayers, which are often considered as simplified models for biological membranes, have been extensively studied by a variety of experimental and theoretical methods (see e.g recent reviews [1-5]).

Polyelectrolytes, being charged polymers under physiological conditions, are nowadays widely used in pharmacy and medicine as carriers for drug and gene delivery, as components of vaccines, etc. [6]. Polypeptide chains (such as antimicrobial peptides and their synthetic analogues) provide another example of how polyelectrolytes affect biological membranes [7]. RNA molecules are also discussed nowadays as promising agents able to prevent gene expression of desease-related proteins [8]. Also, probably the most important biological macromolecule, DNA, is a strongly charged polyelectrolyte. A distinguishing feature of polyelectrolyte systems is that their physical properties are often totally dominated by the electrostatic interactions [9]. That requires their special treatment both in analytical theoretical description of polyelectrolytes and in their computer simulations.

There exists a substantial number of studies concerning either polyelectrolyte conformations [10-13] or the interactions between electrically charged surfaces [14-18]. However, the number of works on interactions between a polyelectrolyte and a charged surface representing biological membrane or colloid particle is still limited. Moreover, most of these studies assume a surface being uniformly charged with no explicit treatment of mobile surface charges which are present in real membranes. In a liquid crystalline state of a bilayer the lipid molecules diffuse freely within the plane. This demixing of the lipids and formation 
of domains in lipid membranes has been observed experimentally [19] and was also studied in computer simulations. It was shown that the lateral movement of lipid molecules plays an important role for membrane systems, e.g. in polyion [20] and colloid [21] adsorption. Adsorption of a single and negatively charged polyion onto a surface carrying both negative and positive charges representing a charged membrane surface has been investigated by Monte Carlo simulations [20], which showed that adsorption appeared even at a net neutral surface or at a weakly negatively charged one. One of the main reasons for this enhanced adsorption is the presence of mobile surface charges forming charged surface patches. Further development of this model was presented in paper [21] where the adsorption of colloids onto a membrane surface was investigated. In that work mobile membrane charges were given an opportunity to perform both lateral movements and also vertical protrusions out of the bilayer. One of the conclusions of work [21] is that surface polarization is more important than protrusions in establishing an attraction between a charged colloid and the net neutral membrane composed of cationic and anionic lipids.

The major phospholipid in mammalian cell membranes is phosphatidylcholine which has a zwitterionic headgroup. To the best of our knowledge there are no works concerning simulation of polyelectrolyte-membrane systems where mobile membrane charges are presented by dipoles. Our aim is an investigation of properties of the model polyelectrolyte-membrane system. The model consists of a polyelectrolyte chain with explicit counterions and of two membrane surfaces carrying mobile dipoles. We allow lateral movement of the dipoles within the membrane plane in order to look for correlation effects between the charged polyion and membrane charges representing the lipid headgroups. Volume of the system is varied during the simulation by changing the distance between the membrane surfaces.

Osmotic pressure is a natural characteristic of the effective forces acting between molecules and an important thermodynamic quantity which can be evaluated experimentally. In recent years several theoretical studies were devoted to calculations of the pressure in the case of polymer-surface interactions, e.g. in semipermeable shell in a solution of polyions [22], in star and comb polymer-membrane systems [23]. In some recent studies of polyelectrolyte-surface interactions two surfaces are present in a model and an uncharged polymer or polyelectrolyte is confined between them [24, 25] or grafted to them [26]. In this case a net force acting upon the surfaces or an osmotic pressure as a function of the distance between them is a particular issue of interest. 
There are different ways of evaluating the pressure in a simulation. For example, in work [27] the osmotic pressure was calculated from the thermodynamic relation: $P_{\text {osm }}=$ $-(\partial F / \partial V)_{T}$ for the system of hexagonally ordered DNA molecules. Here $F$ is the Helmholtz free energy of the system and in order to obtain the pressure, free energy differences of the system at different volumes should be evaluated.

When addressing problems related to conformational properties of complex macromolecules, conformational transitions or thermodynamical properties, standard modeling techniques face difficulties due to problems with sampling of the configuration space. In such situations the approaches based on the generalized ensembles techniques, entropy sampling, Wang-Landau (WL) algorithm [28-31] were proved to be efficient. In the present work we use entropic sampling (ES) method within Wang-Landau algorithm. This approach unlike conventional MC methods allows to estimate the density of states in a single MC run via a random walk which produces a flat histogram in the energy and/or volume space. The resultant density of states can be used to produce canonical averages for calculation of thermodynamic quantities at arbitrary temperature and volume. The advantages of this method over other techniques are that it permits us to access directly the free energy and entropy, is independent of temperature and allows to calculate temperature and volume dependencies of various characteristics of the given chain in a wide temperature and volume range.

In recent years a number of works concerning investigation and modification of WLalgorithm appeared where issues of the convergence of the algorithm [32], dependencies of the CPU time and the error [33] on the simulation parameters were discussed. In order to increase efficiency of the method different schemes of changing the modification factor and number of MC steps in an iteration were proposed [34-37]. Other modifications of the method are associated with the limitation of the energy range accessible for the model by setting up higher and lower boundaries allowed for the energy depending on the temperature range taken into consideration [36, 38, 39]. In recent work [36] two steps WL-procedure have been proposed. During the first step the energy density of states is estimated. The second step is a production run, based on the determined density of states, during which various structural and energetical quantities are sampled [36]. If one is interested in low temperature properties and in obtaining the ground states of the system, for example, global update of the density of states can be used [40]. However, there still exist many questions concerning efficient simulation scheme within WL-algorithm. 
Most of the calculations performed within WL approach deals with the density of states as a function of only one variable, usually energy. In recent paper [41] such variable was volume, or distance between two equally charged plates with electrolyte primitive model in between. However, in some cases it is useful to calculate the joint density of states of two variables, for example, energy and volume, because it allows one to obtain free energy dependence on temperature and volume or eventually pressure. Obviously, the latter case is much more time consuming because the significant number of bins is necessary to cover all the necessary energy and volume range. Recently proposed global update technique [40] speeds up the calculations of the joint density of states by automatically pushing a random walker to the states with low probabilities of realization in the statistical ensemble. However, it is a challenge to obtain a two-dimensional density of states in a wide range of energies and volumes even for a moderate size system.

The plan of the paper is as follows. In Sec. 2 we discuss the model of the polyelectrolytemembrane system. Sec. 3 presents the simulation method, i.e. the general idea of entropic sampling within the WL-algorithm and some modifications of the method. The obtained results and their treatment are given in Sec. 4. Sec. 5 contains final remarks.

\section{MODEL}

In this study we consider a continuous model of a polyelectrolyte-membrane system. A membrane is presented by two plane surfaces, perpendicular to $\mathrm{Z}$ axis and holding mobile dipoles. A system of strongly charged polyion accompanied by neutralizing counterions is placed between the membrane surfaces. The polyion is presented as a flexible chain which consists of $N$ successive beads (monomers) of unit diameter. We consider polyions with $N=8,16,24$. Each monomer of the chain is a hard sphere which carries a unit negative electric charge. Because the counterions of the opposite sign are necessarily present in any real system to provide electroneutrality, an appropriate number of them is added. Each mobile ion is represented by a hard sphere of unit diameter and charge. A dipole of membrane is presented by two ions of unit diameter and opposite unit charges which are always confined to the surfaces. These dipoles mimic zwitterionic head groups of lipids. Centers of the dipole ions are separated by the constant unit distance so that their surfaces are in touch. In order to satisfy the excluded volume condition, the beads of the polyion chain cannot overlap (self- 
avoiding polymer). That is also true for the mobile ions and membrane dipoles. However, as long as we use the WL-algorithm for our simulations, self-intersections of the chain, overlaps of the mobile ions and dipoles are allowed in the course of simulations, and are then accounted for in a proper way, as it was done in our previous paper [42]. The details of this procedure are described in the next section.

The volume of the simulation cell is $V=L^{2} z$, where $L$ is the side length of the cell in $X Y$ directions and $z$ is its height. The volume of the main cell is varied during the simulation by shifting one of the surfaces along Z-axis. Periodic boundary conditions are imposed along X and $\mathrm{Y}$ axes. In our simulations $L$ is constant and equals to 7 . The maximum value of $z$ is taken to be equal to $N-1$ while the minimum value depends on the number of monomers in the polyion as well as on the numbers of counterions and dipoles and is determined during the simulation.

Note that in our simulations the length of the polyion exceeds the simulation box size in $X Y$ directions, so that the polyion can in some configurations contact a periodic image of itself. This can be interpreted as modeling of a finite polyion concentration when periodic images mimic neighboring polyions.

Polyion monomers, membrane dipoles and mobile ions interact by Coulombic potential $V\left(r_{i j}\right)=q_{i} q_{j} / \varepsilon r_{i j}$, where $q_{i}$ and $q_{j}$ are charges, $\varepsilon$ is the relative static permittivity of the solvent, and $r_{i j}$ is the distance between the charges. The natural units for our model are the charge $q\left(q_{i}= \pm q\right)$ and the monomer diameter $d$ which is equal to the diameter of a counterion and of a dipole ion $(d=1)$. So the energy is measured in $\frac{q^{2}}{\varepsilon d}$ units and the temperature in $\frac{q^{2}}{\varepsilon d k_{B}}$. In the following, we shell consider reduced temperature and set therefore $k_{B}=1$. Remembering the definition of the reduced polyion charge density $\xi$ as the ratio of the Bjerrum length (distance at which the energy of interaction of unit charges is equal to the thermal energy) to the charge spacing along the polyion, we can conclude that $\xi=1 / T$ in the adopted units. Thus the correspondence is established between the reduced temperature $T$ of our model and the parameter $\xi$, the characteristic of real polyelectrolytes.

A correct treatment of the long-range electrostatic interactions is of great importance for simulations of electrically charged systems. It is generally accepted that the Ewald summation $[43,44]$ is the most consistent way to treat them, although the possibility of artifacts due to artificial perodicity introduced by the Ewald summation is still debated [45]. As it was shown in our previous work [42] for certain polyelectrolyte systems the 
minimum image approach gives results practically coinciding with the Ewald summation, especially for longer chains, simulated with a larger number of ions, where the screening is stronger. It coincides with the results of Ref. [20] where test simulations of polyion adsorption onto membrane surfaces were performed using minimum image convention and gave results essentially indistinguishable from those with the Ewald summation. Note also that the minimum image convention provides local electroneutrality and explicitly includes the interactions between all the particle pairs in the system, which removes in fact most of shortcomings of the simple spherical electrostatic cutoff scheme with small cutoff distance that is sometimes used in biomacromolecular simulations. That is why we use the minimum image approach in our simulations.

The surface concentration of dipoles is an important parameter which affects their mobility and correspondingly the forces emerging between membrane surfaces and polyelectrolyte. Therefore we carry out two series of calculations for different concentrations of dipoles. The area of the membrane surface in the main cell equals $S_{x y}=L \times L=49$ in both series. The number of dipoles $N_{d p l}$ is equal to 10 in the first series and $N_{d p l}=15$ in the second one. If we define surface concentration of dipoles as the ratio between surface covered by dipoles $S_{\text {cov }}$ to the XY-size of the main cell $S_{x y}$ we can write

$$
n_{d p l}=\frac{S_{c o v}}{S_{x y}}=\frac{2 N_{d p l} \cdot \pi(d / 2)^{2}}{S_{x y}}=\frac{\pi N_{d p l}}{2 \cdot 49}
$$

Thus for the first series of calculations $n_{d p l}=0.32$ and for the second one $n_{d p l}=0.48$.

\section{METHOD}

Density of states $\Omega(E, V)$ for the investigated system can be used to compute canonical averages as functions of volume and temperature by simple integration. Free energy and entropy up to a constant can be obtained from the density of states as well. Finally osmotic pressure is calculated as a derivative of Helmholtz free energy: $P_{\text {osm }}=-(\partial F / \partial V)_{T}$. Thus our primary task is to obtain $\Omega(E, V)$.

For this purpose we use entropic sampling [29, 30] within the WL algorithm [31] approach, with modifications developed in our previous works $[42,46,47]$ aiming to treat polymer systems efficiently. We consider a configurational space of the system consisting of all possible configurations, including overlapping ones. Allowing the system to go through overlapping 
configurations is essential for fast sampling of different regions of the configuration space otherwise separated by high free energy barriers. Thus all possible configurations can be divided on overlapping and non- overlapping ones. Each nonoverlapping configuration can be characterized by a certain energy $E$, which we assume to be in the range between $E_{\min }$ and $E_{\max }$, and the volume $V$ in the range $V_{\min } \leq V \leq V_{\max }$. These ranges of energies and volumes are divided into a finite number of equal small intervals $\left(I_{E}\right.$ and $I_{V}$ intervals of size $d E$ and $d V$ for energy and volume correspondingly), so that each nonoverlapping configuration belongs to one of them. Thus we have a $2 \mathrm{D}$ area which consists of $B_{E V}=I_{E} \cdot I_{B}$ small squares corresponding to all possible pairs of energy and volume. In our calculations the number of such bins $B_{E V}$ ranges approximately from 1300 to 5700 . The number of bins $B_{\Omega}$ which were actually visited during the simulation is slightly less than $B_{E V}$. Values $E_{\min }$, $E_{\max }, V_{\min }, V_{\max }, I_{E}, I_{V}, B_{E V}, B_{\Omega}$ for the different polyion lengths and concentrations of the dipoles are presented in Table 1.

Actually, since very high energy states are not important for calculating canonical properties at finite $T>0$, the boundary of detailed division into energy boxes $E_{\max }$ for each $V$ is chosen to be lower than the maximum energy possible in principle. For all nonoverlapping states with energy $E>E_{\max }$ and with a fixed volume a single bin is assigned. Minimal possible energy $E_{\min }$ is determined in the simulations.

As in previous work [42], another set of bins is introduced for overlapping configurations. We sum overlaps of the polyion's beads and free ions with each other and with the dipoles. Intersections of the dipoles are not allowed. The degree of penetration of the polyion's beads and free ions behind the moving membrane surface is determined as the difference of $z$ coordinates of the surface and of the particle and is added to the number of overlaps. Penetration of the particles behind the unmovable membrane surface (fixed at $z=0$ ) is not allowed.

In principle, we can use one single bin for all overlapping states and get its normalized weight during calculation. However, as it was clearly shown in our previous work [46], such an approach is not stable, and hence it is necessary to introduce a scheme with a set of bins for overlaps. Each such bin corresponds to configurations with a certain number of overlaps for a fixed volume. The number of bins describing overlapping for each $V$ depends on the length of the polyion and is taken to be in the range $30-100$. Every possible number of overlaps less than this boundary value is associated with a separate bin, and one additional 
TABLE I: Parameters of simulations for the different polyion lengths and number of dipoles.

\begin{tabular}{|c|c|c|c|c|c|c|c|c|c|c|c|c|}
\hline & $E_{\min }$ & $E_{\max }$ & $d E$ & $I_{E}$ & $V_{\min }$ & $V_{\max }$ & $d V$ & $I_{V}$ & $B_{E V}$ & $B_{\Omega}$ & $B_{\text {over }}$ & $B_{\text {tot }}$ \\
\hline \hline$N=8, N_{d p l}=10$ & -33.5 & -10.5 & 1 & 24 & 72.5 & 342.5 & 5 & 55 & 1320 & 1303 & 1714 & 3017 \\
\hline$N=8, N_{d p l}=15$ & -45.5 & -10.5 & 1 & 36 & 92.5 & 342.5 & 5 & 51 & 1836 & 1774 & 1632 & 3406 \\
\hline$N=16, N_{d p l}=10$ & -39 & 19 & 2 & 30 & 115 & 735 & 10 & 63 & 1890 & 1886 & 4136 & 6022 \\
\hline$N=16, N_{d p l}=15$ & -51 & 19 & 2 & 36 & 135 & 735 & 10 & 61 & 2196 & 2193 & 4036 & 6229 \\
\hline$N=24, N_{d p l}=10$ (mobile) & -42.75 & -0.25 & 0.5 & 86 & 175 & 735 & 10 & 57 & 4902 & 4766 & 6053 & 10819 \\
\hline$N=24, N_{d p l}=10$ (fixed) & -40.25 & -0.25 & 0.5 & 81 & 205 & 735 & 10 & 54 & 4374 & 4181 & 5968 & 10149 \\
\hline$N=24, N_{d p l}=15$ & -52.75 & -0.25 & 0.5 & 106 & 205 & 735 & 10 & 54 & 5724 & 5561 & 5901 & 11462 \\
\hline
\end{tabular}

bin is used for the number of overlaps exceeding it. The number of bins for overlapping configurations $B_{\text {over }}$ approximately ranges from 1600 to 6000 . Thus the total number of bins in our simulations $B_{t o t}$ varies approximately from 3000 to 11000 . Values $B_{\text {over }}$ and $B_{\text {tot }}$ are presented in Table 1 as well.

Each configuration of the simulated system can have either a definite number of overlaps or, if no overlaps occur, would have definite energy and volume. Thus any configuration of our system can be attributed to one of the bins of the first or second type. For the completely random sampling, the share of nonoverlapping conformations decreases with the increase of the length of the polyion and of the concentration of the dipoles, and for the largest system considered in our work, $N=24$ and $N_{d p l}=15$, falls to about $10^{-15}$.

We perform a random walk in the whole configurational space, allowing overlaps, according to the WL procedure. As long as we implement two-dimensional ES-WL sampling algorithm the density of states $\Omega$ depends on two parameters: energy (or number of overlaps) and volume. Index $i$ corresponds to the first parameter (energy or the number of overlaps) and $j$ - to the latter one (volume). As in previous works [42, 46, 47], it is convenient to introduce the entropy distribution $S_{i j}=\ln \Omega_{i j}$ (initially all $S_{i j}=0$ ). Two sets of counters are introduced: one accumulates $S_{i j}$ for each bin (entropy counter); another one, $n_{i j}$, counts visits to the corresponding states yielding at the end of the run the normalized visit probabilities $p_{i j}=\frac{n_{i j}}{\sum_{i j} n_{i j}}$ (the histogram). The aim is to achieve a flat distribution, $p_{i j}$, over all the states.

A MC step includes a standard trial change of the state with a uniform coordinate 
distribution. For our polyelectrolyte-membrane model, four kinds of steps are performed: we modify the chain's conformation, move a mobile ion, move a dipole within the membrane surface or move one of the membrane surfaces itself (a random shift along $\mathrm{Z}$ axis). The other surface has a fixed position at $z=0$ during the simulations. In order to change the position of a randomly chosen ion we make a shift along one of the axes $\mathrm{X}, \mathrm{Y}$ or $\mathrm{Z}$. In order to change the conformation of the chain we perform steps of three different types:

1. We choose homogeneously one of the beads from 1 to $N-1$ (e.g. the $k$-th) and change randomly the directions of the chain link between beads $k$ and $k+1$. The remaining piece undergoes a parallel shift. As long as we do not intend to change the energy of the system strongly in a single step, the trajectory displacement is made this way small.

2. We choose homogeneously one of the beads from 2 to $N-1$ (e.g. the $k$-th) and rotate it randomly around the direction connecting the beads $k-1$ and $k+1$ (the so called crankshaft move).

3. Reptation of the chain is also used: we cut off a segment from one end of the chain (which is chosen randomly) and build a new one at the opposite end in a random direction.

Changes of positions of randomly chosen dipoles were performed by steps of the following three types:

1. The dipole is shifted along $\mathrm{X}$ and $\mathrm{Y}$ axes.

2. Random rotation of a dipole.

3. Flip of the dipole's charges.

In order to clarify the role of mobile dipoles on the surface of membrane, some simulations were carried out with dipoles fixed at the surface. It such a case, the dipoles were located in a regular manner, with favorable (plus to minus) orientations of neighbouring dipoles to each other.

The trial steps are accepted with the following transition probability condition [31]:

$$
p\left(i j \rightarrow i^{\prime} j^{\prime}\right)=\min \left[1, \exp \left(S_{i j}-S_{i^{\prime} j^{\prime}}\right)\right] .
$$


If this condition is fulfilled, the trial state $\left(i^{\prime} j^{\prime}\right)$ is accepted, and in the opposite case the accepted one is the initial state $(i j)$. Finally, the entropy of the accepted state $\left(S_{i j}\right.$ or $\left.S_{i^{\prime} j^{\prime}}\right)$ is augmented by $\Delta S$, and the corresponding counter of visits $\left(n_{i j}\right.$ or $\left.n_{i^{\prime} j^{\prime}}\right)$ is increased by 1 .

A series of such elementary steps constitute a sweep. According to the original WLalgorithm [31], at the end of a current sweep $\Delta S$ had to be decreased: $\Delta S \rightarrow c \Delta S$ by a factor $0<c<1$ (in [31] $c=0.5$, and the initial value $\Delta S=1$ were used). After several sweeps the $S_{i j}$ dependence is formed and finetuned in the whole range of configurations. At the same time, the probability distribution of visits, the histogram $p_{i j}$, becomes flat, with probabilities approximately equal to the inverse of the number of bins. Under conditions that the increment $\Delta S$ is negligibly small, and the histogram $p_{i j}$ is flat, the accumulated $S_{i j}$ values correspond to the microcanonical entropy, and $\Omega_{i j}$ to the density of states at given values $\left(E_{i}, V_{j}\right)$.

In our previous work [42] we used factor $c=0.9$ which resulted in increasing number of series required to reach small enough $\Delta S$ values (about 130 series vs 25 series corresponding to $c=0.5)$. At the same time such slow decreasing of $\Delta S$ provided more stable sampling of the energy space and allowed us to obtain canonical properties of the system for the low energy area. In the present study we are interested in the behaviour of the system occurring at effective temperatures close to $T=1$. That is why we use a more economic scheme with $c=0.7$, where the value of $\Delta S$ decreases from 0.1 to $8 \cdot 10^{-7}$ through 34 series.

In the present work we use a two stage procedure to obtain $\Omega_{i j}$. The first stage is the standard WL approach with $c=0.7$ which is described above. The second one is used to obtain more accurate distribution $\Omega_{i j}$. During the second stage we calculate the correction to $\Omega_{i j}$ which properly takes into account the deviation of the probability distribution of visits from the uniform one. We perform a random walk satisfying the transition probability condition (2) and take $\Delta S=0$. Unlike the first stage the entropy of accepted states $S_{i j}$ (and therefore the distribution $\Omega_{i j}$ ) now remains intact. Counting of the visits $n_{i j}$ is performed as usual. Second stage approximately takes the same amount of MC steps as the first one and at the end of it we obtain the histogram of visits $p_{i j}$ which is close to the uniform one but is not completely flat. Entropic sampling method can be considered in our case as a simulation in the microcanonical ensemble expanded by two parameters: energy $E$ and volume $V$. We employ here the idea presented in [28] for the expanded ensemble method: the natural logarithm of the ratio of visits to two different subensembles is added to the calculated 
entropy. Thus we analogously add the natural logarithm of probability distribution of visits to the entropy:

$$
S_{i j} \rightarrow S_{i j}+\ln p_{i j}
$$

This way we take into account the deviation of the histogram of visits $p_{i j}$ from the uniform one and thus refine the distribution $S_{i j}$. Since at the second stage $\Delta S=0$, conditions for the Markov chain are strictly satisfied, which allows to avoid problems related to saturation of statistical error, and evaluate accuracy of simulations by running a few simulations of the same system using different sets of random numbers [48].

During the first stage the number of steps in our simulations starts from 100000 in the first series and is multiplied by $1 / \sqrt{\Delta S}$ in every following one. Of course, it is desirable if the system visits every bin during each series. In our case this condition can not be fulfilled within a reasonable time due to the complexity of the system under investigation. That is why we require that the system visits at least $70 \%$ of bins during a specific series. Since we decrease $\Delta S$ quite slowly the landscape of entropy $S_{i j}$ is smoothed down in following series. The number of steps for the second stage of calculations is of the same order of magnitude as for the first one and we apply additional condition that the $99 \%$ of the bins visited during the first stage are visited on the second stage. The bins which are not visited at the second stage do not take part in the calculation of canonical properties.

There exists a well known problem of getting out of the state which was found at one of the later sweeps [42]. When the quantity of $\Delta S$ is already small it takes a long time to accumulate large enough entropy to provide a reasonable acceptance probability (2) to leave the state. Here we introduce an improved procedure to decrease the number of steps necessary for the system to leave such a state and continue visiting the other ones. After each sweep we subtract the lowest entropy $S_{\min }=\min _{i j}\left(S_{i j}\right)$ from every value of $S_{i j}$ :

$$
S_{i j} \rightarrow S_{i j}-S_{\min }
$$

This procedure keeps the entropy difference between old and newly visited states minimal and helps system to sample states more efficiently. At the same time the procedure does not change the $\Omega_{i j}$ landscape itself.

During simulation we determine the entropy $S_{i j}$ corresponding to each bin, which can 
then be converted into the normalized density of states $\Omega\left(E_{i}, V_{j}\right)$ taken over nonoverlapping configurations only:

$$
\Omega\left(E_{i}, V_{j}\right)=\frac{\exp \left(S\left(E_{i}, V_{j}\right)\right)}{\sum_{\text {nonoverlap }} \exp \left(S\left(E_{i}, V_{j}\right)\right)}
$$

The distribution $\Omega\left(E_{i}, V_{j}\right)$ obtained within the WL procedure can be used for calculating canonical properties, i.e. conformational energy, heat capacity, entropy, free energy etc, over wide temperature and volume ranges according to standard relations, e.g. for the internal energy we get:

$$
\langle E\rangle\left(T, V_{j}\right)=\frac{\sum_{i} E_{i} \exp \left(-\frac{E_{i}}{T}\right) \Omega\left(E_{i}, V_{j}\right)}{\sum_{i} \exp \left(-\frac{E_{i}}{T}\right) \Omega\left(E_{i}, V_{j}\right)} .
$$

$\left\langle E^{2}\right\rangle$ is calculated in the same way, yielding the heat capacity as a function of temperature for a fixed volume:

$$
C\left(T, V_{j}\right)=T^{-2}\left(\left\langle E^{2}\right\rangle\left(T, V_{j}\right)-\left(\langle E\rangle\left(T, V_{j}\right)\right)^{2}\right) .
$$

In the expressions (5) and (6) the normalization of $\Omega\left(E_{i}, V_{j}\right)$ is not important, since it enters both numerator and denominator in (5) and any constant factor cancels.

Osmotic pressure is calculated as a derivative of Helmholtz free energy:

$$
P=-(\partial F / \partial V)_{T}
$$

As we are interested in the derivative of the free energy an additive constant to $F$ is not important and we can compute $F$ as:

$$
F\left(T, V_{j}\right)=-T \ln \sum_{i} \Omega\left(E_{i}, V_{j}\right) e^{-\frac{E_{i}}{T}}
$$

The two-dimensional density of states $\Omega(E, V)$ allows one also to compute averages in NPT-ensemble for any given pressure $P$ and temperature $T$, making averaging with the additional factor $e^{-P V / T}$. For any physical property $A$ the NPT-ensemble average can be computed as:

$$
\langle A\rangle(T, P)=\sum_{i, j} A_{i j} \Omega\left(E_{i}, V_{j}\right) e^{-\frac{E_{i}}{T}} e^{-\frac{P V_{j}}{T}}
$$


where $A_{i j}$ is the computed during simulation average value of A taken over configurations corresponding to given bin $\{i j\}$. For example, average volume corresponding to given pressure $P$ can be determined:

$$
V(T, P)=\sum_{i, j} V_{j} \Omega\left(E_{i}, V_{j}\right) e^{-\frac{E_{i}}{T}} e^{-\frac{P V_{j}}{T}}
$$

Equation (10) represents an alternative way to obtain $P(V)$ dependence. In the present work, we used the both ways to compute the osmotic pressure.

\section{RESULTS}

The basic result of our WL-calculations is the density of states $\Omega(E, V)$, which enables us to obtain canonical averages in a wide temperature range for different volumes. The distributions $\Omega(E, V)$ for the polyelectrolyte-membrane system with $N=24$ and $N_{d p l}=$ 10, 15 are presented in Figs. 1(a) and 1(b). As one can see, the WL procedure allows, in a single simulation, to reach states in the $(E, V)$ plane having densities (probabilities) differing by factor more than $10^{30}$.

In Figs. 2(a) and 2(b) the isotherms $E(V)$ for different temperatures are shown for the system with $N=24$ and $N_{d p l}=10,15$, for fixed and for mobile dipoles at the surfaces. $z$ denotes distance between the membrane surfaces which is proportional to the volume. All the curves have a similar shape. Starting from the smallest volume the energy abruptly falls and reaches its minimum. Comparing cases of fixed and mobil dipoles, we can see that on small distances the energy is lower for the case of mobile dipoles. This is because the mobile dipoles can favorably adjust their positions interacting with charged polyion. At large distances between the surfaces, the interaction with polyion is less important, and system with fixed dipoles has lower energy (remembering that the dipoles were fixed in favorable orientation with respect to each other). The values of energy are lower for the lower temperatures. For the case $N_{d p l}=15$ the minimum of energy is shifted to the left with the increase of temperature while for $N_{d p l}=10$ (mobile dipoles) it is observed at approximately the same volume for all $T$. After reaching their minima the isotherms start to ascend and the energy increases faster for higher temperatures. For $N_{d p l}=10$ the $E(V)$ curves reach higher energies than for $N_{d p l}=15$.

The dependencies of the specific energy of the system with $N=24$ and $N_{d p l}=10,15$ 


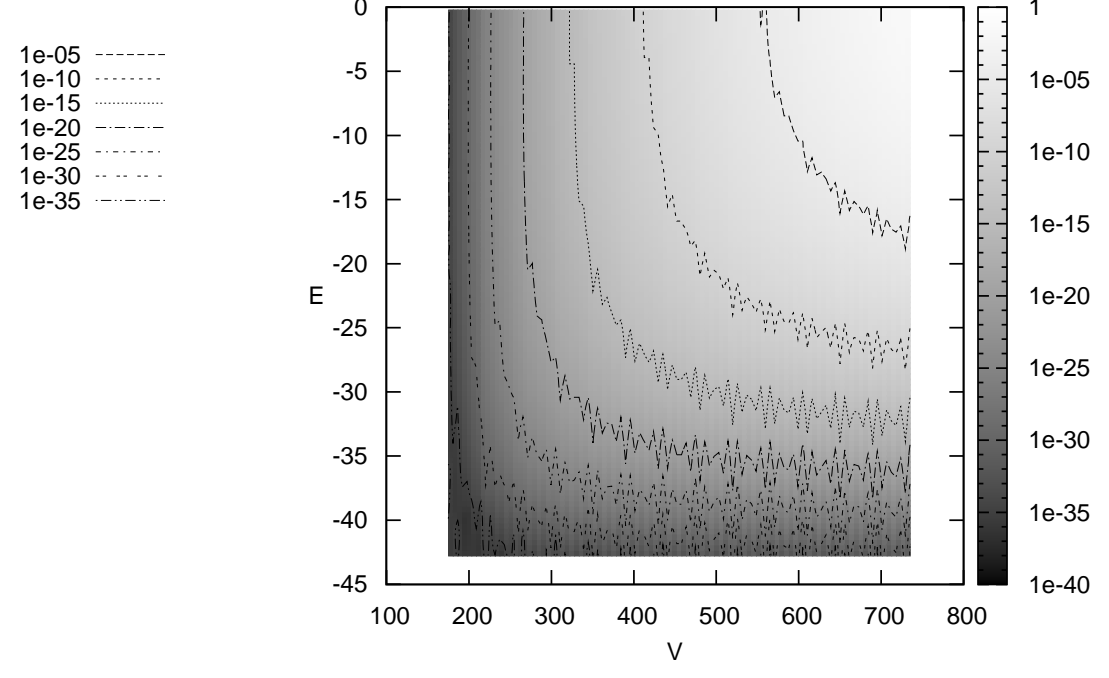

(a)

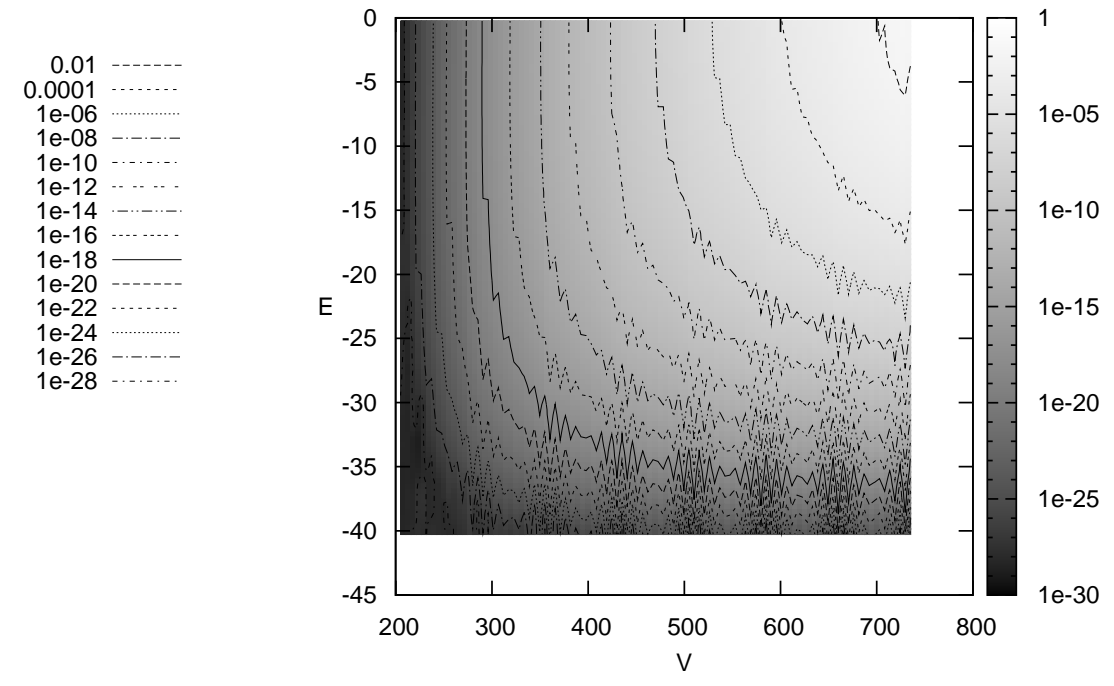

(b)

FIG. 1: The density of states $\Omega(E, V)$ for the system with $N=24$. (a) $N_{d p l}=10$, (b) $N_{d p l}=15$.

on temperature, isohores $E(T)$, obtained from eq. (5) for different volumes, are presented in Figs. 3(a) and 3(b). The curves show similar behaviour, with energy reaching a low asymptotic value (practically accessible energy of the ground state) at $T<0.2$, and gradual 


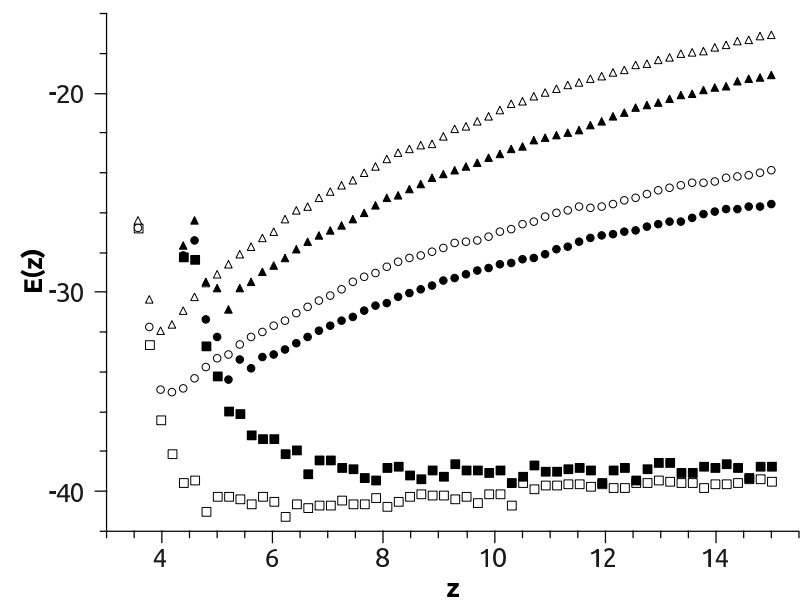

(a)

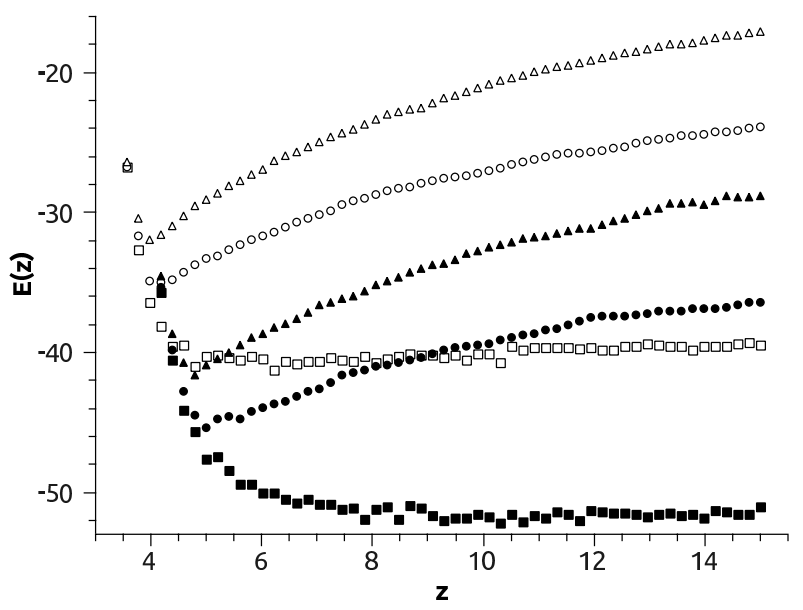

(b)

FIG. 2: The isotherms $E(z)$ for different temperatures for the system with $N=24$. Squares, circles and triangles stand for $T=0.2 ; 0.6 ; 1.0$ correspondingly. $z$ denotes distance between the membrane surfaces. (a) $N_{d p l}=10$, empty symbols correspond to mobile dipoles and filled - to fixed ones. (b) Comparison of cases $N_{d p l}=10$ and $N_{d p l}=15$, mobile dipoles (empty and filled symbols corresponingly)

growth of energy at higher temperatures. Similar behaviour was observed in our previous study of unconfined lattice polyelectrolyte [42] Comparison of simulations with fixed and mobile dipoles (Fig. 3a) shows that at low temperatures lower energies can be reached in the case of mobile dipoles. In the case of higher concentration of the dipoles $N_{d p l}=15$, Fig. 3(b), the asymptotic value of energy at low temperatures is lower than for the case $N_{d p l}=10$. With the increasing of temperature the curves corresponding to greater volumes raise steeper and achieve higher levels of energy.

The dependency of heat capacity on temperature at constant volume, $C_{V}(T)$, is often used as one of the ways to determine the temperatures areas of phase transitions. In Figs. 4(a) and 4(b), temperature dependencies of heat capacity are presented for the system with $N=24$ and $N_{d p l}=10 ; 15$, as well as for the cases with fixed and mobile dipoles at the surfaces. The obtained $C_{V}(T)$ isohores look similar to the corresponding curves for a free lattice polyelectrolyte [42]: the functions tend to zero at low and high temperatures, have a tall peak at their left side and a long high-temperature shoulder. For lager separation between the surfaces $(z=15)$, the heat capacity maximum is moved to temperature about 0.2 , that is the same value which was observed for free polyelectrolyte in paper [42]. 


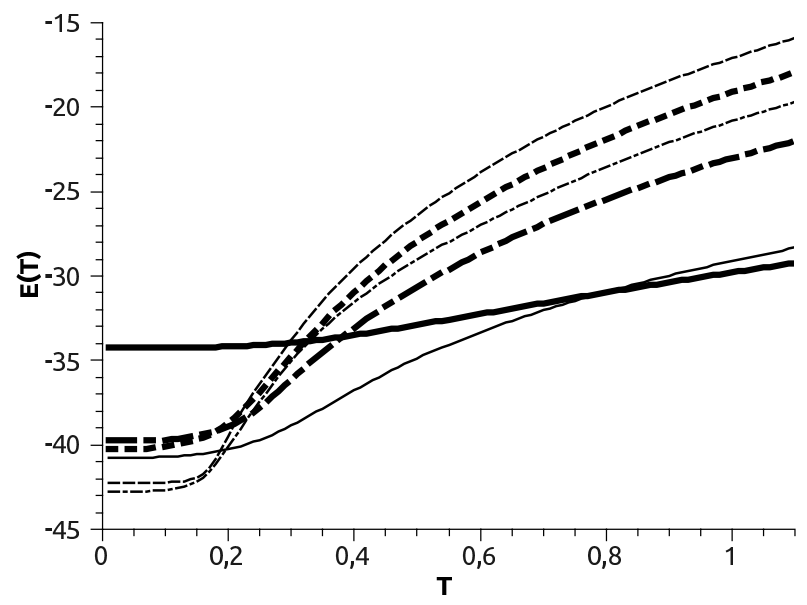

(a)

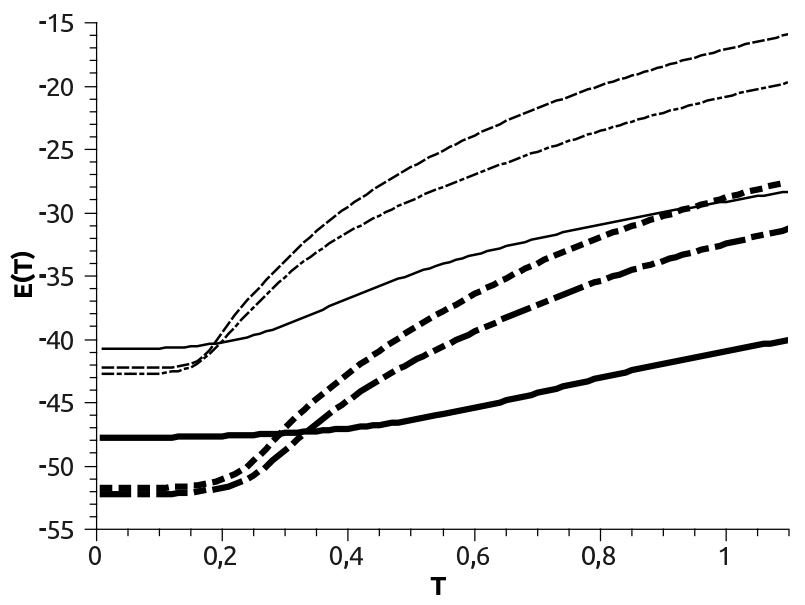

(b)

FIG. 3: The dependencies of the specific energy of the system with $N=24$ on temperature. $z=5.0,10.1,15.0$ (solid, dash-dot and dash lines) are distances between the membrane surfaces. (a) $N_{d p l}=10$, thin lines correspond to mobile dipoles, thick lines - to fixed ones. (b) Comparison of cases $N_{d p l}=10$ and $N_{d p l}=15$ (thin and thick lines respectivelyly).

It was demonstarted previously [40] that too coarse discretization of energy within the WL algorithm can lead to inconsistent calculations of temperature dependent thermodynamics properties, for example heat capacity may acquire false peaks. For the system with $N=24$ and 10 mobile dipoles we have made a few test computations with different descretization of energy, taking $d E$ equal to 2,1 , and 0.5 , obtaining very similar result for the last two values. From this we concluded that the observed peaks of heat capacity reflect the real behaviour.

The investigations of coil-globule, liquid-solid and other transitions of a single uncharged homopolymer have been made within Wang-Landau algorithm, for example, in papers [4951]. It is concluded that for the homopolymer high temperature peak (or shoulder) on $C_{V}(T)$ curve corresponds to coil-globule transition and the high peak at low temperature - to the liquid-solid one. Nevertheless, for the polyelectrolyte-membrane system electrostatic interactions play crucial role so an equivalence of transition areas with the single uncharged polymer should not be expected, and studies of typical conformations, radius of gyration, correlation functions along with heat capacity are needed for characterization of the transition.

In Figs. 5(a-d) we present the volume dependencies of the canonical part of the free energy $F$. The dependencies $F(z)$ play a role of the potential of mean force between the 


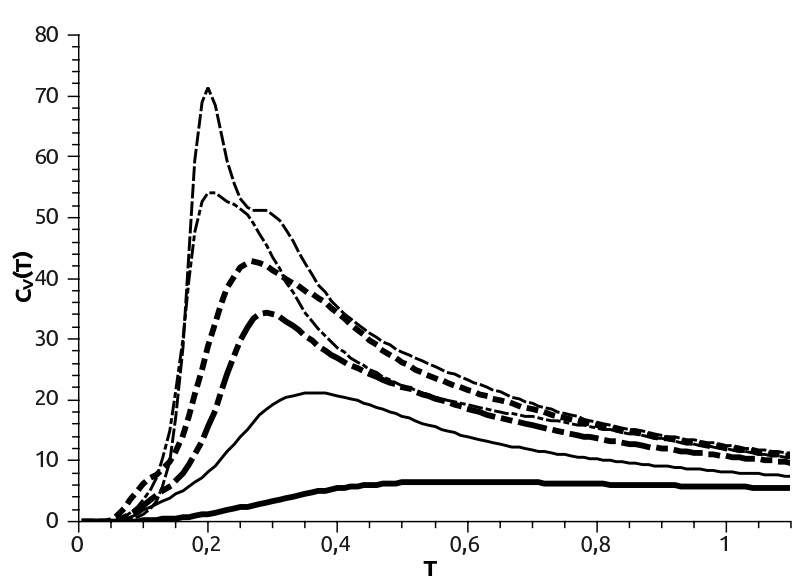

(a)

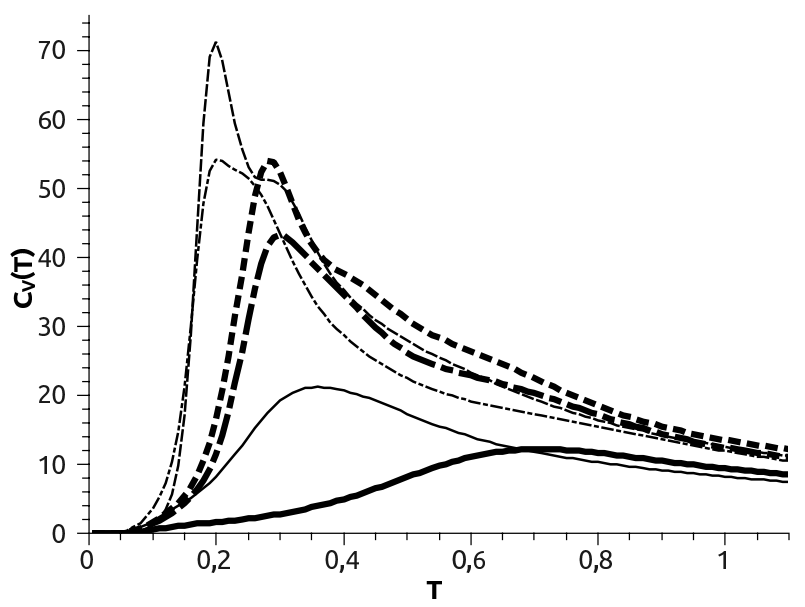

(b)

FIG. 4: Temperature dependencies of heat capacity $C_{V}(T)$ for the system with $N=24$. $z=$ 5.0,10.1, 15.0 (solid, dash-dot and dash lines) are distances between the membrane surfaces. (a) $N_{d p l}=10$, thin lines correspond to mobile dipoles, thicklines - to fixed ones. (b) Comparison of cases $N_{d p l}=10$ and $N_{d p l}=15$ with mobile dipoles (thin and thick lines respectively).

surfaces, and can be used also for calculation of the osmotic pressure by numerical differentiation.

Comparing $E(z)$ (Figs. 2(a) and 2(b)) and $F(z)$ (Figs 5(a-d)) curves, one can note that while the energy often has a minimum at some distance between the surfaces, the free energy curves do not have such one. That is, despite the presence of energy minimum, the interaction between the surfaces in all cases considered here is repulsive, evidently due to strong entropy effects. Our test calculations showed, that only by choosing very low temperatures, below 0.07, one can in some cases obtain a minimum at the free energy curve. Such low values of the reduced temperature are however not relevant for real membranes and polyelectrolytes in aqueous solutions.

Another observetion concerns the role of mobility of dipoles at the surface. Fig. $5 \mathrm{~d}$ demonstrates, that while at larger distances between the surfaces the mobility of dipoles does not play a major role for the interaction (free energy) between the surfaces, at small distances the systems with fiexd dipoles show stronger repulsion. Thus one can conclude that mobility of dipoles at the surfaces gives attractive contribution to the total force between the surfaces, though this attraction cannot overcome the overall repulsion.

In Figs. 6(a-d), volume dependencies for osmotic pressure for all considered polyion 


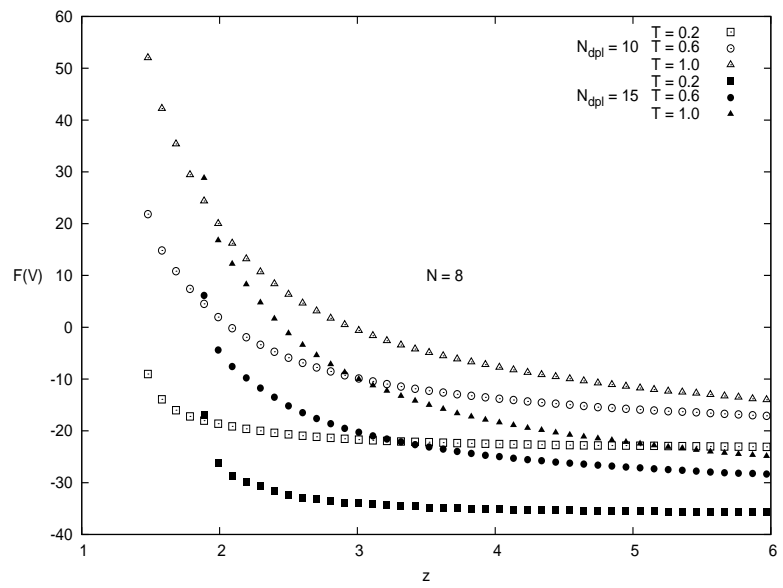

(a)

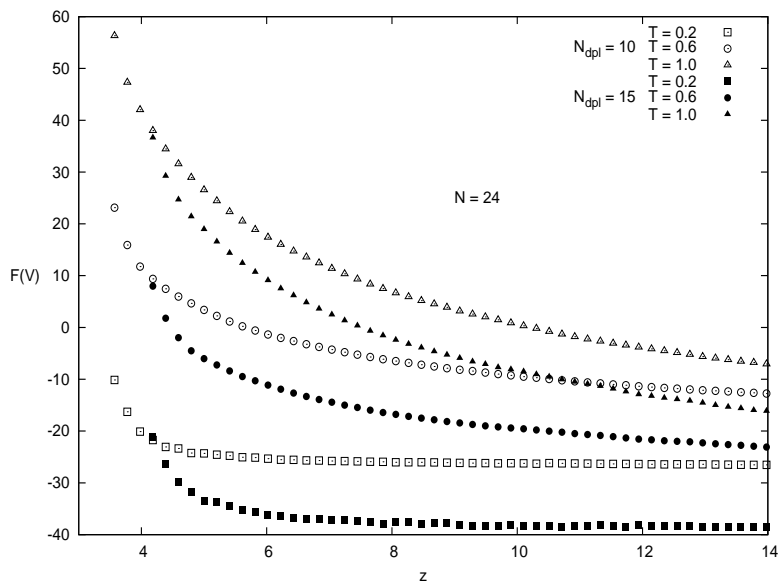

(c)

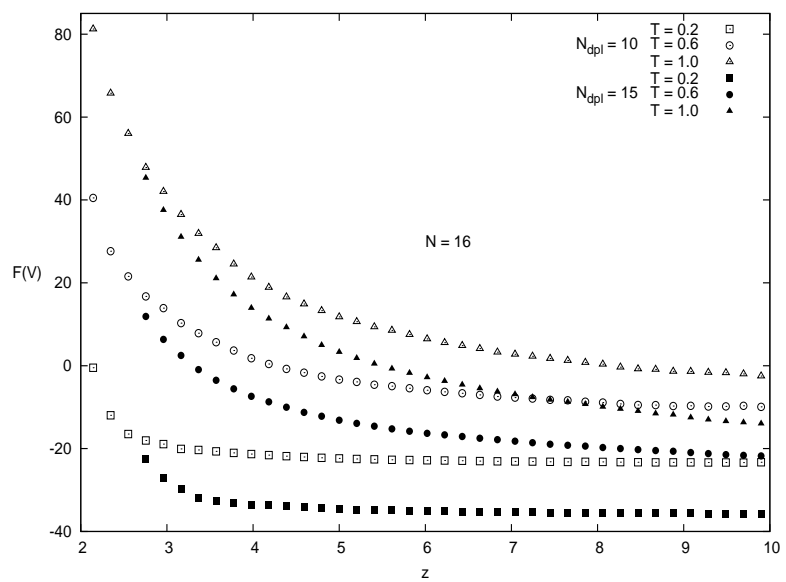

(b)

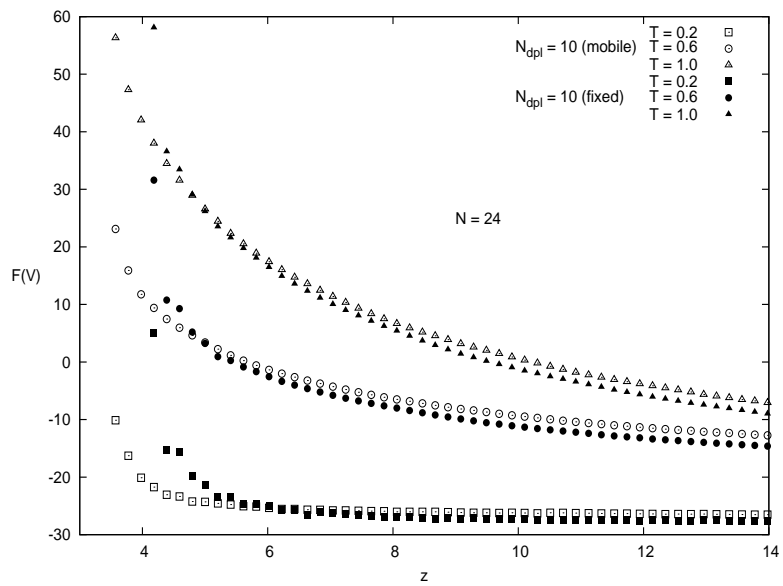

(d)

FIG. 5: Volume dependencies of the canonical part of the free energy $F$ for the system with $N=8,16,24$. Squares, circles and triangles stand for $T=0.2 ; 0.6 ; 1.0$ respectively. $z$ denotes distance between the membrane surfaces. (a) $N=8$, (b) $N=16$, (c) and (d) $N=24$. (a),(b),(c) Empty symbols correspond to the case $N_{d p l}=10$, filled ones - to $N_{d p l}=15$ (mobile dipoles in both cases). (d) $N_{d p l}=10$, empty symbols correspond to mobile dipoles and filled - to fixed ones.

lengths and dipoles concentrations are presented. Two sets of data are given for each case: osmotic pressure calculated by numerical differentiation of the free energy is shown as points, and osmotic pressure obtained by inverting $V(P)$ curve calculated in NPT ensemble by e.q. (10) is shown as lines. One can see that two ways of calculation of osmotic pressure produce very similar results. In all considered cases the osmotic pressures are positive and gradually decrease to zero at large distances. The decrease of temperature leads to lower osmotic 
pressure, or to less repulsive interaction between the surfaces.

In Fig. 6(a), which represents the case with the polyion consisting of 8 monomers one can clearly see two vertical asymptotes corresponding to the two different concentrations of the dipoles in the membrane surfaces. These vertical asymptotes correspond to the minimal possible separation between the membrane surfaces, when further decrease of the separation distance becomes impossible due to steric hindrances. For lower concentration the monomers can seemingly set themselves between the dipoles and the system can contract to the lowest volume. The vertical asymptotes are the same for different temperatures but the pressure is higher for the highest $T$. In the Figs. 6(b-d) corresponding to the polyion lengths 16 and 24 there also exist vertical asymptotes which are located in the area of larger $z$. It can be explained by the existence of the excluded volume which increases with the increasing of the number of monomers in the chain.

In Fig. 6(e), osmotic pressure between membrane surfaces with mobile dipoles, but in ansense of polyion and mobile ions is shown. One can see that in such case, the pressure is weakly negative, which corresponds to attraction at small distances between the surfaces. This attraction, arising due to dipole fluctuations, has the same origin as Wan-der-Waals effect, and it was demonstrated previously for the case of two surfaces containing mobile dipoles and separated by dielectric media, by perturbation calculations and direct Monte Carlo simulations [52].

While NVT and NPT description seems to give consistent data for $P(V)$ isotherms, an interesting question may be how large volume fluctuations one can expect under condition of constant (osmotic) pressure. Volume fluctuations are related also to the compressibility of the system. Distribution over volumes at given pressure is easily obtained from the free energy curve as:

$$
\rho(V)=e^{-\frac{F(V)}{T}} e^{-\frac{P V}{T}}
$$

Several such distributions are shown in Figs. 7(a) and 7(b). One can see that distribution over volumes becomes broader for lower pressures. Appearance of several maxima in the volume distributions at low temperatures is another feature. It may correspond to different modes of folding of the polyion between the membrane surfaces.

We have also estimated possible statistical uncertainty by running simulations for $N=24$, with 10 mobile dipoles three times by changing each time sequence of random numbers. For 


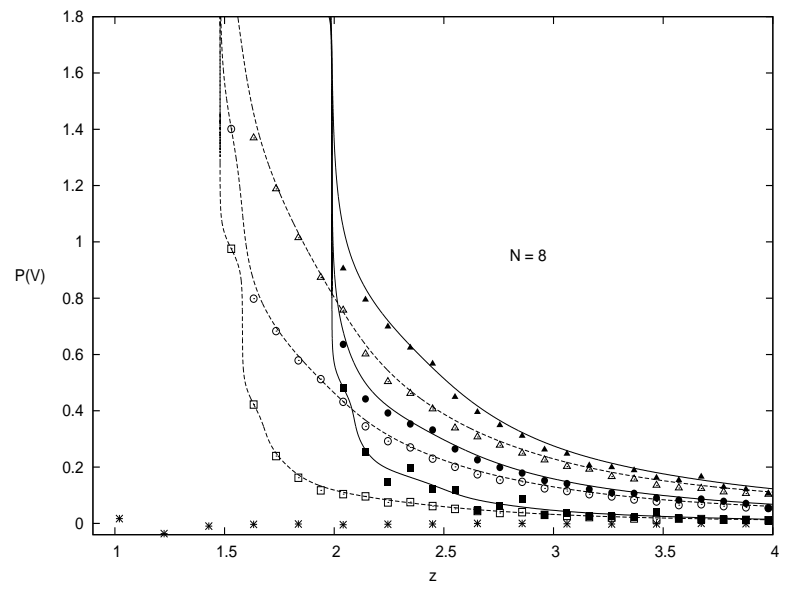

(a)

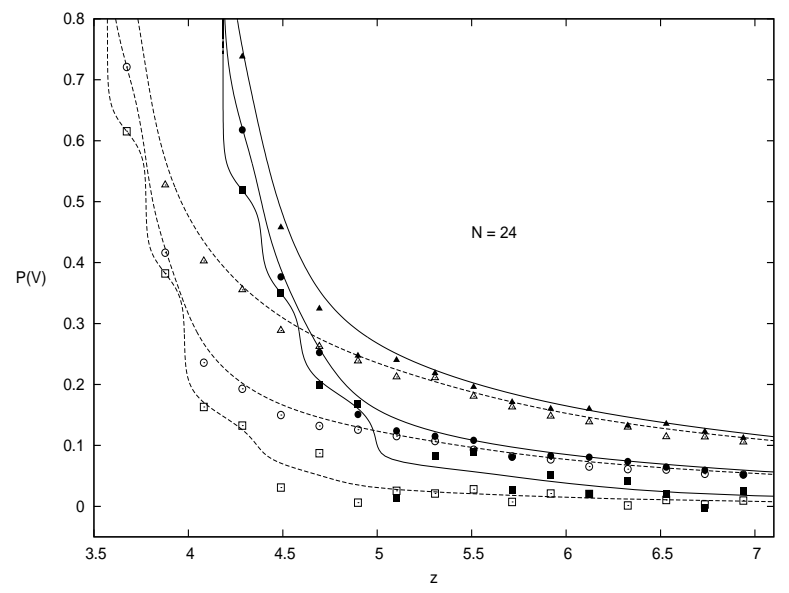

(c)

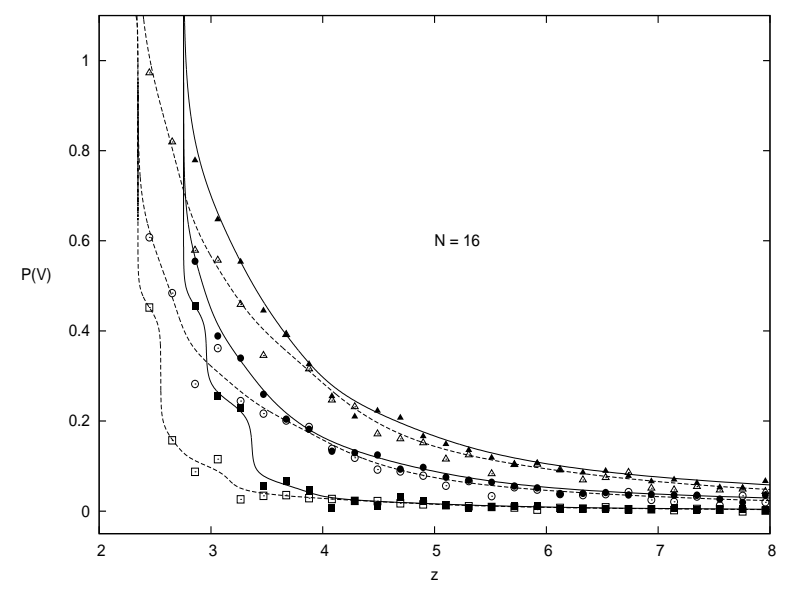

(b)

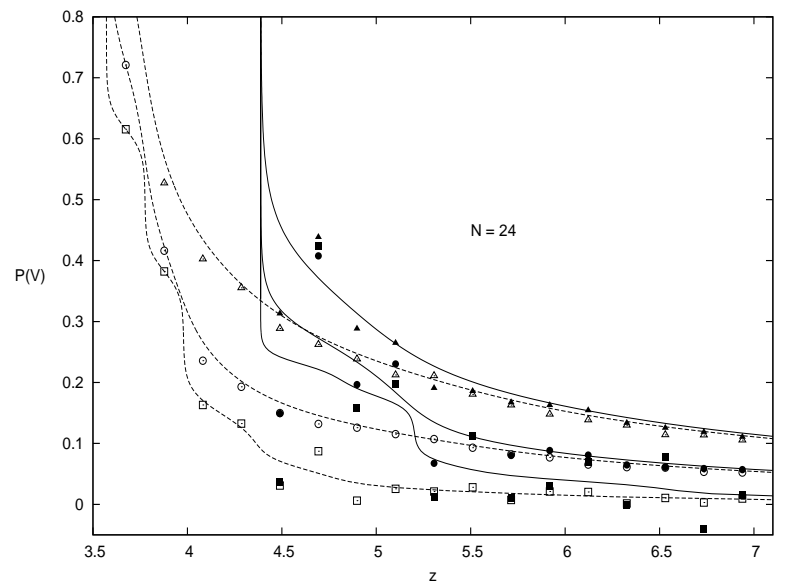

(d)

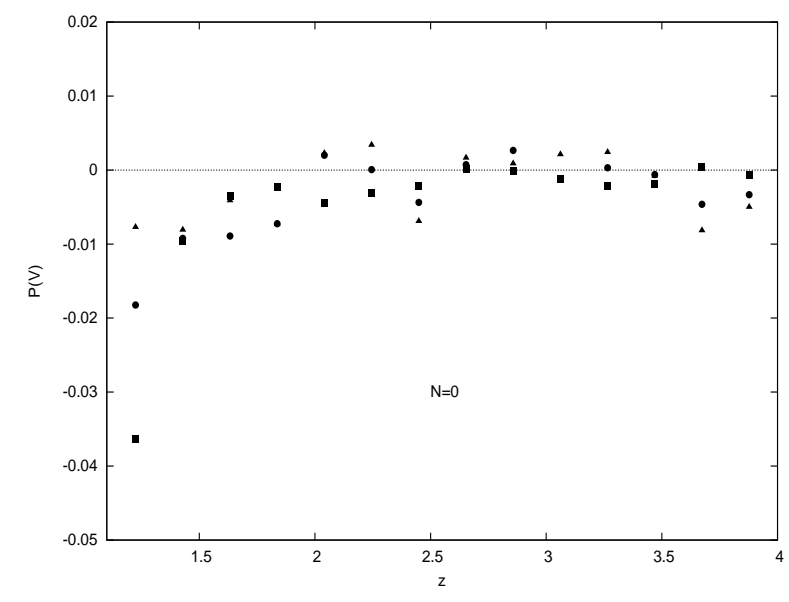

(e) 
FIG. 6: Volume dependencies for the osmotic pressure, $P(V)$. (a) $N=8$, (b) $N=16$, (c) and (d) $N=24$, (e) $N=0$. Symbols stand for the data obtained from Helmholtz free energy, curves - from Gibbs energy. Squares, circles and triangles stand for $T=0.2 ; 0.6 ; 1.0$ respectively. (a-c) Empty symbols and dash curves: $N_{d p l}=10$, filled symbols and solid curves: $N_{d p l}=15$ (mobile dipoles in both cases); (d) $N_{d p l}=10$, empty symbols and dash curves: mobile dipoles, filled symbols and solid curves: fixed dipoles. (e) case when only membrane surfaces with mobile dipoles are present with $N_{d p l}=15$

most of properties we did not observed any noticeable difference in results. Some difference between the three runs was observed for the energy and free energy (no more than 5 energy units) at small distances between the surfaces $(Z<6)$, and for low (below 0.2) temperature (again, a few energy units). As it is seen from the scale of corresponding quantities (Figures 2-5), these uncertainties do not bring any qualitative difference in the behaviour of the curves.

\section{CONCLUSIONS}

In this paper we extended our previous ES-simulations of polymer and polyelectrolyte models within WL-algorithm [42, 46, 47] applying it now to a continuous model of a polyelectrolyte between membrane surfaces. Within two-dimensional ES-WL sampling algorithm we

obtained joint density of states $\Omega(E, V)$ as a function of energy and volume in a single run. Various properties of the system over wide temperature and volume or pressure ranges, i.e. conformational energy, heat capacity, free energy, were obtained from $\Omega(E, V)$ by simple integration (summation). Finally osmotic pressure was calculated as a derivative of Helmholtz free energy, and from inverting $V(P)$ dependence computed in the NPT-ensemble.

The advantages of the used scheme are the nearly uniform sampling of all possible states according to WL probability of transitions and that overlaps of polyion monomers, counterions, dipoles are not rejected but are accounted for in a proper way which results in better sampling of rare configurations of the system and easier barrier crossing. In order to increase efficiency of our calculations we introduced a number of modifications to original WL-approach, i.e. expanded ensemble corrections to the density of states $\Omega(E, V)$ at the 


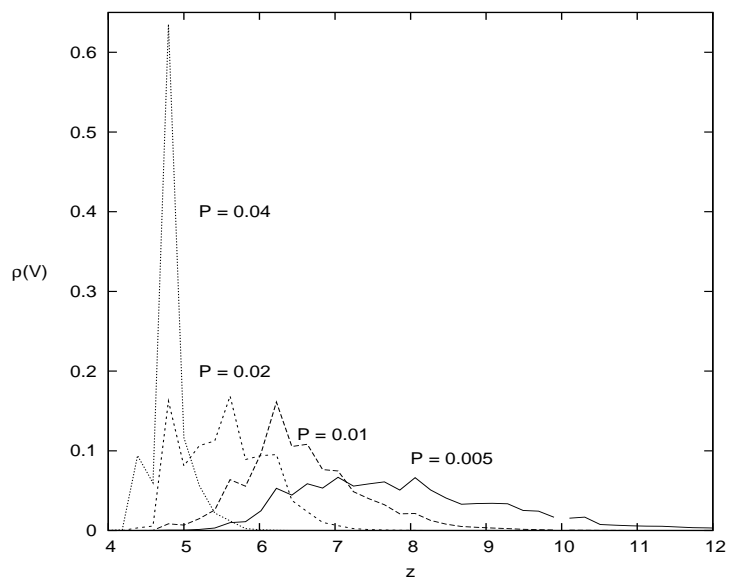

(a)

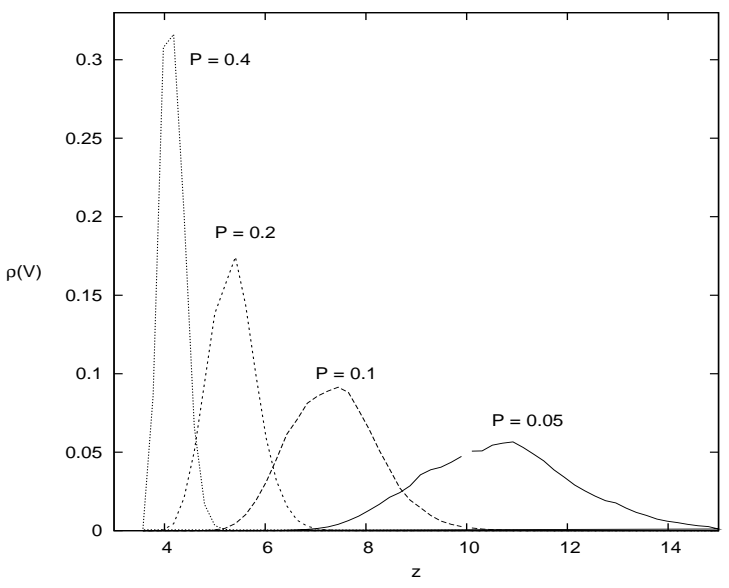

(b)

FIG. 7: Distribution over volumes $\rho(V)$ for $N=24, N_{d p l}=10$, where $z$ denotes distance between the membrane surfaces. (a) $T=0.2$, (b) $T=1.0$.

final stage of calculations and a way to avoid being "stuck" in a newly found states by extracting the lowest entropy from $S(E, V)$ distribution.

Another advantage of the used two-dimensional WL scheme is that the Helmholtz free energy is obtained as a function of volume. In "normal" one-dimensional WL method at constant volume [31], one can determine the free energy by coupling the system with some reference state of known free energy, as it was done in our previous paper [42]. This is not necessary within the present approach, since the free energies computed for different volumes, have the same additive constant which disappears upon derivation.

The presented two-dimensional ES-WL sampling scheme can be readily applied for studies 
of equilibrium thermodynamic properties of even more complicated and realistic models giving an opportunity to obtain canonical averages in NVT and NPT ensembles. In order to investigate phase transitions in the system different configurational properties, e.g. radius of gyration, end-to-end distance for the polyion etc., can be calculated as well.

Our calculations have been done for different polyion lengths and concentrations of dipoles on the membrane surfaces. What may seem somewhat surprising in the results, is that we did not observe appearance of effective attraction between such surfaces in any of the studied cases. It was demonstrated on many examples that oppositely charged surfaces may attract each other due to the presence of oppositely charged multivalent ions or polyions between them $[14,25,41]$. In average neutral surfaces containing mobile dipoles attract each other due to well known van-der-Waals (London) effect[52]. A flexible polyion surrounded by counterions contracts when the reduce temperature falls down below 1 and finally collapses to a globular state at reduced temperature about $0.2[42,53]$. In our systems we observed only decrease of repulsion between the surfaces at lower temperatures. A plausible explanation of this may be that our system contains too many mobile components (mobile dipoles, ions, and flexible polyion), and decrease of entropy upon contraction or cooling of such system has a larger role compared to the case when dipole surfaces, or a polyion are taken separately of each other.

\section{Acknowledgments}

The work has been supported by the Swedish Institute Visby Programme, by the Swedish Research Council (Vetenskapsrådet, grant no. 621-2007-5256), and by Russian Foundation

for Fundamental research (RFFI) grant 02-08-0004/a. Computational facilities have been provided by the Center for Parallel Computing (PDC), Stockholm.

[1] S.J.Marrink, A.H. de Vries and D.P.Tieleman, Biochim. Biophys. Acta, Biomembr., $\mathbf{2 0 0 9 , 1 7 8 8 , 1 4 9 .}$

[2] E.L.Elson, E.Fried, J.E.Dolbow and G.M.Genin, Annu. Rev. Biophys., 2010, 39, 207.

[3] Y. Teramura and H. Iwata, Soft Matter, 2010, 6, 1081.

[4] A.J.Garcia-Saez and P. Schwille, FEBS Lett., 2010,584, 1653. 
[5] A.P.Lyubartsev and A.L.Rabinovich, Soft Matter, 2011, 7, 25.

[6] A. V. Kabanov and T. Okano Adv. Exp. Med. Biol. 2003, 519, 1.

[7] M. Zasloff, Nature (London) 2002, 415, 389.

[8] L. Melnikova, Nature Review Drug Discovery, 2007, 6, 863.

[9] A: P. Lyubartsev and L. Nordenskiöld, In: Handbook of polyelectrolytes and their applications", Am. Sci. Publ. Part 3., 2002, 309-326.

[10] M. J. Stevens and K. Kremer, J. Chem. Phys., 1995, 103, 1669.

[11] R. R. Netz, Phys. Rev. Lett. 2003, 90128104.

[12] A. Naji and R. Podgornik, Phys. Rev. E 2005, 72041402.

[13] P. Y. Hsiao and E. Luijten, Phys. Rev. Lett. 2006, 97, 148301.

[14] R. Kjellander, T. Åkesson, B. Jönsson and S. Marcelia, J. Chem. Phys., 1992, 97, 1424.

[15] F. Otto and G. N. Patty, J. Chem. Phys., 2000, 112, 8939.

[16] J.-P. Hansen and H. Löwen, Annu.Rev.Phys.Chem., 2000, 51209.

[17] R. Kjellander, A. P. Lyubartsev and S. Marcelja, J. Chem. Phys., 2001, $114,9565$.

[18] D. Ben-Yaakov and D. Andelman, Physica A - Stat.Mech. and its Appl. 2010, 3892956.

[19] B Maier, J. O. Rädler, Macromolecules 2000, 33, 7185.

[20] R. S. Dias, A. A. C. C. Pais, P. Linse, M. G. Miguel, and B. Lindman, J. Phys. Chem. 2005, 109, 11781 .

[21] R. S. Dias, P. Linse , Biophys. Journ. 2008, 94, 3760.

[22] R. Tsekov, M. R. Stukan, O. I. Vinogradova, J. Chem. Phys. 2008, 129, 244707.

[23] T.-Y. Wang, C.-M. Fang, Y.-J. Sheng, H.-K. Tsao, J. Chem. Phys.,2009, 130,124904.

[24] A. Broukhno, B. Jönsson, and T.Åkesson, J. Chem. Phys., 2000, 113, 5493.

[25] M. Turesson, C. E. Woodward, T. Åkesson, J. Forsman, J. Phys. Chem. B, 2008, 112, 5116.

[26] O. J. Hehmeyer, M. J. Stevens, J. Chem. Phys., 2005, 122, 134909.

[27] A. P. Lyubartsev, L. Nordenskiöld, J. Phys. Chem., 1995, 99, 10373.

[28] A. P. Lyubartsev, A. A, Martsinovskii, S. V. Shevkunov, and P. N. Vorontsov-Velyaminov, J. Chem. Phys., 1992, 96, 1776.

[29] B. A. Berg and T. Neuhaus, Phys. Rev. Lett. 1992, 68, 9.

[30] J. Lee, Phys. Rev. Lett. 1993, 71, 211.

[31] F. Wang and D. P. Landau, Phys.Rev. Lett., 2001, 86, 2050.

[32] C. Zhou and R. N. Bhatt, Phys. Rev. E, 2005, 72, 025701. 
[33] H. K. Lee, Y. Okabe, D. P. Landau, Computer Physics Communications, 2006, 175, 36.

[34] P. Poulain, F. Calvo, R. Antoine, M. Broyer, and P. Dugourd, Phys. Rev. E, 2006, 73, 056704.

[35] R. E. Belardinelli and V. D. Pereyra, Phys. Rev. E, 2007, 75, 046701.

[36] C. Gervais, T. Wüst, D. P. Landau, and Y. Xu, J. Chem. Phys. 2009, 130, 215106.

[37] A. N. Morozov and S. H. Lin, J. Chem. Phys., 2009, 130, 074903.

[38] M. S. Shell, P. G. Debenedetti, and A. Z. Panagiotopoulos, Phys. Rev. E, 2002, 66, 056703.

[39] A. Tröster and C. Dellago, Phys. Rev. E, 2005, 71, 066705.

[40] C. Zhou, T. C. Schulthess, S. Torbrugge, and D. P. Landau, Phys. Rev. Lett. 2006, 96, 120201.

[41] J. Zelko, A. Iglic, V. Kralj-Iglich, and P. B. S. Kumar, J. Chem. Phys., 2010, 133, 204901

[42] N. A. Volkov, P. N. Vorontsov-Velyaminov, A. P. Lyubartsev, Phys. Rev. E, 2007, 75, 016705.

[43] P. Ewald, Ann. Phys., 1921, 64, 253.

[44] M. P. Allen, and D. J. Tildesley, "Computer Simulations of Liquids", 2nd ed. Clarendon, Oxford, 1987.

[45] P. H. Hunenberger, and J. A. McCammon, J. Chem. Phys., 1999, 110, 1856.

[46] P. N. Vorontsov-Velyaminov, N. A. Volkov, and A. A. Yurchenko, J. Phys. A: Math. Gen. 2004, 37, 1573.

[47] N. A. Volkov, A. A. Yurchenko, A. P. Lyubartsev, and P. N. Vorontsov-Velyaminov, Macromol. Theory Simul. 2005, 14, 491.

[48] Q.Yan and J. J. de Pablo, Phys. Rev. Lett., 2003, 90, 035701.

[49] D. F. Parsons and D. R. M. Williams, Phys. Rev. E, 2006, 74, 041804.

[50] W. Paul, T. Strauch, F. Rampf, and K. Binder, Phys. Rev. E, 2007, 75, 060801.

[51] D. T. Seaton, T. Wüst, and D. P. Landau, Phys. Rev. E, 2010, 81, 011802.

[52] B.Jönsson, P. Attard and D. Mitchel, J.Phys.Chem., 1988, 92 5001.

[53] C. G. Jesudason, A. P. Lyubartsev and A. Laaksonen, Eur. Phys. J. E, 2009, 30, 341. 